\title{
Nivel de orientación empática en los estudiantes de terapia física y rehabilitación de una universidad estatal
}

\author{
Jorge Luis Cañamero Riquelme ${ }^{1}$, Cristhian Santiago Bazán ${ }^{2}$
}

\section{RESUMEN}

Objetivo: Determinar el nivel de orientación empática en los estudiantes de Terapia Física y Rehabilitación de la Universidad Nacional Mayor de San Marcos, 2016.

Materiales y métodos: Estudio de enfoque cuantitativo de tipo descriptivo-observacional, prospectivo, con diseño no experimental de corte transversal. Realizado en 134 estudiantes de Terapia Física y Rehabilitación del 2do al 5to año de carrera de la Escuela Académico Profesional de Tecnología Médica de la UNMSM; se empleó la Escala de Empatía Médica de Jefferson (EEMJ).

Resultados: En general se obtuvo una puntuación media de $114.60 \pm 14.424$, que corresponde a un nivel alto de orientación empática. Cualitativamente esta categoría está representada por el $85.1 \%$. Respecto a las dimensiones, se obtuvieron puntuaciones medias de “Toma de perspectiva" (61.33, nivel alto [91.0\%]), “Atención por compasión” (41.16, nivel alto [82.8\%]) y “Capacidad de Ponerse en el Lugar del Paciente" (12.38, nivel medio [61.2\%]). No hay diferencias significativas en general ni en las dimensiones según sexo y según edad ( $p>0.05)$, pero sí se encuentran diferencias significativas según año de carrera.

Conclusión: Los estudiantes de Terapia Física y Rehabilitación de la Universidad Nacional Mayor de San Marcos, presentan del 3er, 4to y 5 to año respecto al 2 do año de carrera $(\mathrm{p} \leq 0.05)$ una orientación empática favorable; específicamente se muestran prestos a la comprensión de los sentimientos de los pacientes y en poner atención a las experiencias personales de los pacientes como medio terapéutico.

Palabras clave: Orientación empática; toma de perspectiva; atención por compasión; capacidad de ponerse en el lugar del paciente.

\section{Level of empathic orientation in students of physical therapy and rehabilitation of a state university}

\section{ABSTRACT}

Objective: To determine the level of empathic orientation in students of Physical Therapy and Rehabilitation of Universidad Nacional Mayor de San Marcos (UNMSM), 2016.

Materials and methods: A quantitative descriptive-observational prospective non-experimental cross-sectional study performed in 134 second- to fifth-year students of Physical Therapy and Rehabilitation at Escuela Académico Profesional de Tecnología Médica of UNMSM. The Jefferson Scale of Empathy (JSE) was used.

Results: In general, a mean score of $114.60 \pm 14.424$ was obtained, corresponding to a high level of empathic orientation. This category is qualitatively represented by $85.1 \%$. Regarding the dimensions, mean scores were obtained in "Perspective Taking" (61.33, high level [91.0\%]), "Compassionate Care" (41.16, high level [82.8\%]) and "Standing in the Patient's Shoes" (12.38, mean level [61.2\%]). There were no significant differences neither in general terms nor in gender and age ( $p>0.05)$, but there were significant differences in the year of the career.

Conclusion: Third, fourth and fifth-year students of Physical Therapy and Rehabilitation of Universidad Nacional Mayor de San Marcos have a favorable empathic orientation compared to second-year students ( $\leq \leq 0.05)$. They specifically show willingness to understand patients' feelings and pay attention to patients' personal experiences as therapeutic means.

Keywords: Empathic orientation; perspective taking; compassionate care; standing in the patient's shoes.

1. Licenciado Tecnólogo Médico en Terapia Física y Rehabilitación.

2. Magister en Gestiòn y Conducciòn en Salud. 
Nivel de orientación empática en los estudiantes de terapia

física y rehabilitación de una universidad estatal

\section{INTRODUCCIÓN}

En ciencias de la salud, muchas son las definiciones que se le da al término "empatía», para este estudio y a manera de introducción, nos referimos a la empatía como un aspecto de la personalidad con notable influencia en las relaciones interpersonales, favoreciendo a la "relación con el paciente", siendo ésta un elemento importante dentro de las ciencias de la Salud ${ }^{(1)}$.

Sobre lo expuesto, brindar al paciente un trato empático, resulta de gran utilidad para diseñar o reforzar los procesos terapéuticos y asistenciales en función a las necesidades de las personas, lo cual daría lugar a obtener información de utilidad diagnóstica con el fin de fortalecer la adhesión al tratamiento por parte del paciente ${ }^{(2)}$.

Por ello es necesario conocer la orientación empática de manera progresiva, desde los primeros años de formación hasta culminar la misma. En este período la orientación empática puede pasar por un proceso de fortalecimiento o de desgaste a medida que el estudiante avanza su formación profesional.

A nivel de América Latina, existen evidencias derivadas de investigaciones realizadas en estudiantes y profesionales de medicina, odontología y kinesiología; no obstante, a nivel nacional dicha información relacionada a la formación en terapeutas físicos es escasa. Por esta razón, se hace necesaria realizar estudios específicamente de la orientación empática, a fin de conocer el comportamiento de la misma.

\section{MATERIALES Y MÉTODOS}

Estudio de enfoque cuantitativo de tipo descriptivoobservacional, prospectivo, con diseño no experimental de corte transversal. Realizado en 134 estudiantes de terapia física y rehabilitación del 2 do al 5to año de carrera de la Escuela Académico Profesional de Tecnología Médica de la UNMSM; se empleó la Escala de Empatía Médica de Jefferson (EEMJ).

\section{RESULTADOS}

En la (Figura 1), se observa que la puntuación media general, alcanzó un valor de $114.60 \pm 14.424$, correspondiente a la categoría "Alto".

En la (Tabla 1), se observa el predominio del nivel "Alto" con el $\mathbf{9 1 . 0 \%}$ y $\mathbf{8 2 . 8 \%}$ para la "Toma de perspectiva" y “Atención por compasión” respectivamente; por otro lado, predomina el nivel "Medio" con el 61.2\% para la dimensión "Capacidad de ponerse en el lugar del paciente". Las valoraciones de probabilidad, señalan que dichas diferencias son significativas $(p=0.000)$.

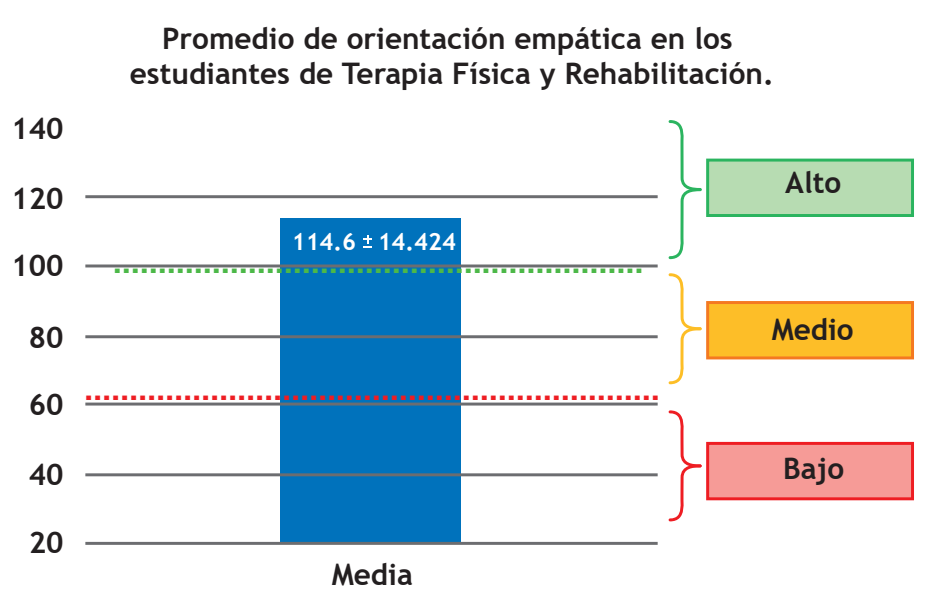

Figura 1. Análisis descriptivo de la orientación empática en general

En la (Tabla 1), se observa el predominio del nivel “Alto" con el 91.0\% y 82.8\% para la "Toma de perspectiva” y “Atención por compasión" respectivamente; por otro lado, predomina el nivel "Medio" con el 61.2\% para la dimensión "Capacidad de ponerse en el lugar del paciente". Las valoraciones de probabilidad, señalan que dichas diferencias son significativas $(\mathrm{p}=0.000)$. 
Tabla 1. Análisis porcentual de los niveles de orientación empática, según dimensiones

\begin{tabular}{|c|c|c|c|c|c|c|}
\hline \multirow[t]{2}{*}{ Niveles } & \multicolumn{2}{|c|}{$\begin{array}{c}\text { Toma de } \\
\text { Perspectiva }\end{array}$} & \multicolumn{2}{|c|}{$\begin{array}{l}\text { Atención por } \\
\text { compasión }\end{array}$} & \multicolumn{2}{|c|}{$\begin{array}{c}\text { Capacidad de } \\
\text { Ponerse en el } \\
\text { Lugar del } \\
\text { Paciente }\end{array}$} \\
\hline & f & $\%$ & $f$ & $\%$ & $f$ & $\%$ \\
\hline Bajo & 1 & 0.8 & 1 & 0.8 & 33 & 24.6 \\
\hline Medio & 11 & 8.2 & 22 & 16.4 & 82 & 61.2 \\
\hline Alto & 122 & 91.0 & 111 & 82.8 & 19 & 14.2 \\
\hline \multirow[t]{2}{*}{ Total } & 134 & 100.0 & 134 & 100.0 & 134 & 100.0 \\
\hline & \multicolumn{2}{|c|}{$\begin{array}{c}x^{2}=201.955 \\
g l=2 \\
p=0.000\end{array}$} & \multicolumn{2}{|c|}{$\begin{array}{c}x^{2}=152.701 \\
g l=2 \\
p=0.000\end{array}$} & \multicolumn{2}{|c|}{$\begin{array}{c}x^{2}=49.000 \\
g l=2 \\
p=0.000\end{array}$} \\
\hline
\end{tabular}

(Tabla 2), se observa datos que fueron sometidos con la finalidad de determinar si existen diferencias significativas entre años de carrera a la prueba ANOVA de un factor, se encontraron diferencias significativas en la orientación empática $(p=0.000)$ y en sus dimensiones "Toma de perspectiva" $(p=0.002)$ y "Atención por compasión" $(p=0.00)$.

Tabla 2. Análisis comparativo de la orientación empática según año de carrera, de manera general y por dimensiones

\begin{tabular}{llllll}
$\begin{array}{c}\text { Año de } \\
\text { carrera }\end{array}$ & Variable & $\mathrm{N}$ & Media & Des. Est. & Prueba estadística \\
\multirow{2}{*}{ 2do año } & Varón & 12 & 107.50 & 11,294 & $\mathrm{t}=0.379 ; \mathrm{gl}=32 ; \mathrm{p}=0.707$ \\
& Mujer & 22 & 105.05 & 20,703 & \\
\multirow{2}{*}{ 3er año } & Varón & 18 & 111.11 & 14.656 & $\mathrm{t}=-2.267 ; \mathrm{gl}=21.633 ; \mathrm{p}=0.034$ \\
& Mujer & 24 & 119.46 & 6.234 & \\
4to año & Varón & 14 & 113.64 & 16.648 & $\mathrm{t}=-1.655 ; \mathrm{gl}=18.863 ; \mathrm{p}=0.114$ \\
& Mujer & 23 & 121.75 & 10,054 & \\
\hline \multirow{2}{*}{ 5to año } & Varón & 11 & 116.55 & 9,331 & $\mathrm{t}=-1.172 ; \mathrm{gl}=19 ; \mathrm{p}=0.256$ \\
\hline
\end{tabular}

En la (Tabla 3), se observa que las valoraciones de probabilidad señalan que dichas diferencias son significativas en el tercer año de carrera $(p=0.034)$, a favor de las mujeres (media $=119.46 \pm 14.656$ ) comparadas con los varones (media=119.46 \pm 6.234). 
Tabla 3. Análisis comparativo de la orientación empática respecto al sexo, según año de carrera

\begin{tabular}{|c|c|c|c|c|c|c|}
\hline & & Variable & $\mathbf{N}$ & Media & Des. Est. & Prueba estadística \\
\hline \multirow{16}{*}{ 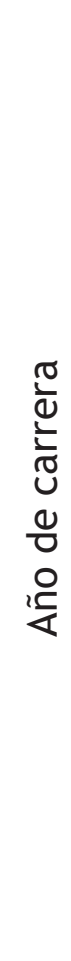 } & \multirow{4}{*}{ Toma de perspectiva } & 2do año & 34 & 56.18 & 11,723 & \multirow{4}{*}{$F=6.301 ; p=0.001$} \\
\hline & & 3er año & 42 & 63.48 & 5,469 & \\
\hline & & 4to año & 37 & 62.65 & 7,432 & \\
\hline & & 5to año & 21 & 63.05 & 5,740 & \\
\hline & \multirow{4}{*}{ Atención por compasión } & 2do año & 34 & 38.26 & 6,912 & \multirow{4}{*}{$F=4.948 ; p=0.003$} \\
\hline & & 3er año & 42 & 40.67 & 6,269 & \\
\hline & & 4to año & 37 & 43.24 & 5,230 & \\
\hline & & 5to año & 21 & 43.14 & 5,209 & \\
\hline & \multirow{4}{*}{$\begin{array}{l}\text { Capacidad de ponerse en el } \\
\text { lugar del paciente }\end{array}$} & 2do año & 34 & 11.76 & 3,239 & \multirow{4}{*}{$F=1.568 ; p=0.200$} \\
\hline & & 3er año & 42 & 11.90 & 3,384 & \\
\hline & & 4to año & 37 & 13.11 & 3,619 & \\
\hline & & 5to año & 21 & 13.05 & 2,559 & \\
\hline & \multirow{4}{*}{ Orientación empática } & 2do año & 34 & 105.91 & 17,796 & \multirow{4}{*}{$F=6.579 ; p=0.000$} \\
\hline & & 3er año & 42 & 115.88 & 11,329 & \\
\hline & & 4to año & 37 & 118.70 & 13,337 & \\
\hline & & 5to año & 21 & 118.90 & 9,762 & \\
\hline
\end{tabular}

\section{DISCUSIÓN}

El estudio se desarrolló en torno a la descripción del comportamiento de la variable Orientación empática en estudiantes de terapia física y rehabilitación de la Universidad Nacional Mayor de San Marcos, esta variable es la resultante de tres dimensiones relacionadas a la Toma de perspectiva, la Atención por compasión, y la Capacidad de ponerse en el lugar del paciente.

En primera instancia se determinó el nivel de la orientación empática de manera general, donde se observa que el promedio total obtenido fue de $114.60 \pm 14.424$, valor que se ubica en la categoría de nivel "Alto".

Estos hallazgos son similares a los de otros investigadores internacionales que encontraron niveles altos de orientación empática como Huberman J. y Colaboradores., mediante su estudio en alumnos de odontología, donde se encontró un promedio de 114.37. A su vez con el de Alonso L., en su estudio en alumnos de medicina, donde encontró una media 107.54. Así mismo, Guadalupe M. y Colaboradores, en su estudio obtienen un promedio de $101.69^{(3,4,5)}$; también investigadores nacionales como Gutiérrez F. y Colaboradores, en su estudio realizado en alumnos de Estomatología, se encontraron niveles de orientación empática altos, con una media de 109.93, concordando así con el presente estudio ${ }^{(6)}$. No obstante, en las investigaciones realizadas por Ñique C. y Torres C., se encontró un promedio de 89.80 y 83,71 correspondientes a niveles medios, resultados que difieren a los obtenidos en este estudio ${ }^{7,8)}$. Las discrepancias con estos últimos estudios podrían deberse, a las diferencias en la malla curricular en las diferentes carreras de la salud.

Seguidamente, se determinó la orientación empática, según las dimensiones antes mencionadas. Se observa que las puntuaciones medias correspondientes a las dimensiones "Toma de perspectiva" (Media: $61.33 \pm$ 8.51) y "Atención por compasión" (Media: $41.16 \pm 6.28$ ) recaen en la categoría de nivel "Alto", y la puntuación obtenida para la dimensión "Capacidad de ponerse en el lugar del paciente" (Media: $12.38 \pm 3.32$ ), recae en el nivel "Medio". Investigadores como Torres C. y Colaboradores encontraron en su estudio, cierta similitud a estos resultados, según las dimensión "Toma de perspectiva" (media $=58,21 \pm 7,54)$ correspondiente con un nivel alto. Sin embargo se encontraron en las dimensiones "atención por compasión" (media $=12,84 \pm 4,76$ ) y "Capacidad para ponerse en el lugar del paciente "(media $=7,42 \pm 3,08$ ) correspondientes a niveles Bajos, resultados que difieren a los obtenidos por este estudio. Otros como Contreras T. 
y colaboradores, encuentran en las dimensiones "Toma de perspectiva" (media $=54,70 \pm 8,10)$, "Atención por compasión" (media $=37,31 \pm 6,86$ ) corresponden a un nivel Alto y la dimensión "Capacidad de ponerse en el lugar del Paciente" (media $=9,57 \pm 3,30$ ) a un nivel medio, similares a los resultados obtenidos en este estudio ${ }^{(8,9)}$.

Por otra parte al comparar la orientación empática, en general y por dimensiones, según sexo de los estudiantes de terapia física y rehabilitación, no se encontraron diferencias significativas, ni en general, ni en las dimensiones ( $p$ > $0.05)$, no obstante, se observa una ligera ventaja de las mujeres sobre los varones, Dichos resultados son similares a los obtenidos por Contreras T. y colaboradores, quienes no encuentran diferencias significativas $(p>0.05)$ de la Orientación empática ni en general ni por dimensiones, según sexo, en estudiantes Kinesiólogos. A su vez Castañeda S. y Parraguez R. en una población similar, no encuentran diferencias significativas $(p>0.05)$ de la orientación empática de manera general, tampoco en dimensiones, según sexo. Concordando así con el estudio ${ }^{(9,10)}$. Por otro lado, investigaciones nacionales como la de Gutiérrez F. y Colaboradores, en estudiantes de estomatología, tampoco encuentran diferencias significativas $(p>0.05)$ de la orientación empática entre varones y mujeres; también Salcedo M. y Díaz V., en estudiantes de odontología, no encuentran diferencias significativas $(p>0.05)$ de la orientación empática de manera general, tampoco en dimensiones, según sexo. Estos resultados son similares a los obtenidos en el presente estudio ${ }^{(6,11)}$.

De la misma manera, al comparar la orientación empática, en general y por dimensiones, según edad de los estudiantes de terapia física y rehabilitación, comparando las valoraciones medias según grupos de edad, no se encontraron diferencias significativas, ni en general ni en las dimensiones $(p>0.05)$.No obstante, cabe resaltar que las puntuaciones obtenidas de la orientación empática en general y por dimensiones tienden a incrementarse a medida que la edad avanza. Los resultados coinciden con el estudio en alumnos de odontología realizado por Ñique $C$, quien no encuentra diferencias significativas $(p>0.05)$. También concuerdan con los de Ubillús G. y Colaboradores, en estudiantes de medicina ${ }^{(7,12)}$.

Estos hallazgos no concuerdan a los de los investigadores Torres C. y Colaboradores, quienes encuentran diferencias significativas $(p<0.03)$ a favor de los estudiantes de fisioterapia más jóvenes ${ }^{(8)}$.

La razón de las diferencias y similitudes de nuestros resultados, a los obtenidos por diversas investigaciones de la orientación empática, según sexo y edad, podría deberse al nivel de desarrollo personal de cada estudiante, experiencias previas durante su carrera y el estado emocional de ellos al momento de haber contestado la EEMJ pudiendo haber influenciado en los resultados.

La comparación de la orientación empática y sus dimensiones, según año de carrera de los estudiantes de terapia física y rehabilitación. De manera general nos muestra diferencias significativas $(p=0.000)$ del 2 do año de carrera con respecto a los demás. Cabe mencionar que los años de carrera más adelantados, tienden a presentar mejores puntuaciones de orientación empática. A su vez, según el año de carrera y dimensión: "Toma de perspectiva" $(p=0.002)$, se observa que los estudiantes de segundo año, están notablemente por debajo de los demás grupos de estudiantes que pertenecen a otros años de carrera; "En la dimensión "Atención por comparación" ( $p=0.003)$; muestra que las puntuaciones medias obtenidas por los estudiantes de 2do año, difieren desfavorablemente, comparados con las puntuaciones obtenidas por los de 4to y 5 to año, más no con las de 3er año, así mismo, las puntuaciones medidas obtenidas por éste grupo (3er año), no difieren significativamente respecto a las puntuaciones obtenidas por los demás años.

En conclusión, en la dimensión "Capacidad para ponerse en los zapatos del paciente" no se encontraron diferencias significativas $(p>0.05)$. Estos hallazgos coinciden con diferentes investigadores como Contreras $T$ y Colaboradores; Castañeda S. y Parraguez R, que encontraron diferencias significativas de $p=0,001$ y $p<$ 0,05 respectivamente, a favor de los estudiantes de años superiores, tanto de manera general y en sus respectivas dimensiones en alumnos de kinesiología ${ }^{(9,10)}$.A su vez los resultados concuerdan con los de nuestros compatriotas Salcedo M y Díaz V., realizados en estudiantes de odontología ${ }^{(8)}$. Por el contrario estos resultados difieren con el estudio de Ubillús G. y Colaboradores, en estudiantes de medicina, quien encontró diferencias significativas, a favor de los estudiantes de años iniciales ${ }^{(12)}$.

La razón de estas diferencias, podría deberse a que en el proceso de formación del terapeuta físico, se incorpora las prácticas lo más temprano posible, generando mayor contacto con el paciente y estableciendo la relación terapeuta físico - paciente. De hecho los estudiantes de 4to y 5 to año de carrera son los que presentan una mayor orientación empática en general y por dimensiones, ya que en esta etapa son pre-internos e internos respectivamente, por lo tanto asumen mayor responsabilidad con el paciente, enfrentándose con mayor madurez y preparación a la atención del paciente.

Con la intención de realizar un estudio exhaustivo de comparación según año de carrera, que plantea la evaluación comparativa según sexo y grupos de edad. Respecto a la evaluación comparativa entre varones y mujeres, solo se ve diferencia significativa en los alumnos del tercer año de carrera, a favor de las mujeres. Por otro lado no hay evidencia que indique diferencias significativas entre los grupos de edad.

\section{REFERENCIAS BIBLIOGRÁFICAS}

1. Hemmerdinger JM, Stoddart S, Lilford RJ. A systematic review of tests of empathy in medicine. BMC Medical Education. 2007; 7:24-32

2. Nouvilas E. Atribución del cumplimiento terapéutico: Diferencias entre pacientes y profesionales de la salud. Estudios de psicología social.2003: 273-280 
Nivel de orientación empática en los estudiantes de terapia

física y rehabilitación de una universidad estatal

3. Silvia M, Arboleda J, Díaz V. Orientación empática en estudiantes de Medicina en una universidad de República Dominicana. Educación Médica Superior. 2014;28(1):74-83.

4. Casas J, Hopp M, Providell S, Narvaez V, Niveles de orientación empática en estudiantes de odontología de la Universidad del Desarrollo, sede Santiago (Chile). Revista Clínica de Periodoncia, Implantología y Rehabilitación Oral. 2014;4(3):169-74.

5. Alonso L, Caro S, Erazo A, Díaz V. Evaluación de la orientación empática en estudiantes de medicina de la Universidad del Norte. Barranquilla (Colombia). Salud Uninorte Barranquilla (Col). 2013; 29(1):22-3.

6. Gutierrez F, Quezada B, Lopez M, Mendez J, Diaz V, Zamorano $A$, et al. Medición del nivel de percepción empática de los estudiantes de la Facultad de Estomatología Roberto Beltrán. Universidad Peruana Cayetano Heredia. Rev Estomatol Herediana. 2012; 22(2):91-9.

7. Ñique-Carbajal C. Orientación empática de estudiantes de odontología de una Universidad Peruana. Kiru. 2013;10(1):4954

8. Torres C, Cadellans A, Martí I. Incremento de la empatía tras la primera interacción de estudiantes de fisioterapia con usuarios de la salud. Madrid: tema libre presentado en II Conferencia Internacional de Comunicación en Salud Universidad Carlos III de Madrid; octubre.2015

9. Contreras T, García S, Guenchugaray L, FernándezA. Orientación Empática de los estudiantes de la carrera de Kinesiología de la Universidad de Magallanes, de la ciudad de Punta de Arenas en el año 2014. Rev Educ Cienc Salud. 2015; 12 (2): 125-339.

10. Rojas-Sarey A, Castañeda-Barthelemiez S, Parraguez-Infiesta R. Orientación empática de los estudiantes de dos escuelas de kinesiología de Chile. EDUC MED. 2009;12(2):103-9
11. Salcedo-Rioja M, Narvaez V. Empatía en estudiantes de odontología de la Universidad Nacional Mayor de San Marcos (Perú). Salud Uninorte Barranquilla (Col). 2015;31(3):565-74.

12. Arriola U, Alcalde S, Kong R, Alcantara R, Ambrosio R, Gomez S. La orientación empática en los estudiantes de medicina humana de la Universidad de San Martin de Porres. Revista Horizonte Médico. 2010;10(2):37-43

Fuentes de financiamiento:

Este artículo ha sido financiado por los autores.

\section{Conflictos de interés:}

Los autores declaran no tener ningún conflicto de interés.

\section{Correspondencia:}

Cristhian Santiago Bazàn

Dirección: Jr. Tiahuanaco 1493 Urb. Zàrate S.J.L

Teléfono: 941703104

Correo electrónico: cristhiansantiagob@gmail.com
Recibido: 04 de enero de 2017 Aprobado: 09 de marzo de 2017 